\title{
Culture Negative Stent Infection in an Infant with Hypoplastic Left Heart and Persistent Fever
}

\author{
Kurt D. Piggott, ${ }^{1}$ Federico Laham, ${ }^{2}$ Alejandro Jordan Villegas, ${ }^{2}$ Harun Fakioglu, ${ }^{1}$ \\ Carlos Blanco, ${ }^{1}$ and Sukumar Suguna Narasimhulu ${ }^{1}$ \\ ${ }^{1}$ The Heart Center at Arnold Palmer Hospital for Children, Pediatric Cardiac Intensive Care, 92 W. Miller Street, MP 307, \\ Orlando, FL 32806, USA \\ ${ }^{2}$ Arnold Palmer Hospital for Children, Pediatric Infectious Disease, USA
}

Correspondence should be addressed to Kurt D. Piggott; kurt.piggott@orlandohealth.com

Received 20 April 2015; Accepted 2 September 2015

Academic Editor: Man-Hong Jim

Copyright (C) 2015 Kurt D. Piggott et al. This is an open access article distributed under the Creative Commons Attribution License, which permits unrestricted use, distribution, and reproduction in any medium, provided the original work is properly cited.

We present an infant with hypoplastic left heart with persistent fever despite two courses of antibiotics and repeatedly negative blood cultures. He eventually underwent surgical extraction of two stents. The stent cultures became positive; he was treated with 4 weeks of antibiotics and the fever resolved.

\section{Background}

Fever is commonly seen in patients following surgery for congenital heart disease. The frequency of fever following cardiovascular surgery has been reported between $12 \%$ and $73 \%$ [1]. A source may not be identified, and if persistent the fever can become especially troublesome [2]. This may be further complicated when the patient has an artificial valve or endovascular stents. Duration of antibiotic therapy is often determined by culture results and clinical status of the patient. We report a case of an infant with hypoplastic left heart and persistent fever who despite numerous negative blood cultures had evidence of stent-associated infection which was confirmed by direct culture after removal. We also discuss the management.

\section{Case Report}

The case is a 4-month-old male born with mitral and aortic stenosis variant of hypoplastic left heart syndrome. He underwent a Norwood procedure with placement of a $4.0 \mathrm{~mm}$ modified Blalock-Taussig shunt. He had a difficult postoperative course plagued by low cardiac output syndrome, vocal cord paralysis, and several failed extubations. One month postoperatively he required cardiac catheterization and placement of a stent in the distal systemic-pulmonary artery shunt due to stenosis and acute desaturation. Two months postoperatively he required stent placement in the interatrial septum due to a progressively increasing gradient by echocardiogram. One month later he was discharged home. He returned 36 hours later with a fever of 106 Fahrenheit (F) and clinical shock. His white blood cell (WBC) count was 21,000 cells $/ \mathrm{mL}$ with a procalcitonin of $212 \mathrm{ng} / \mathrm{mL}$ and c-reactive protein (CRP) of $<0.5 \mathrm{mg} / \mathrm{dL}$. He was pan-cultured and placed on broad-spectrum antibiotics. All cultures remained negative, including viral studies. $\mathrm{He}$ was treated for 14 days with vancomycin and cefepime for presumed culture negative septic shock. He was discharged home 48 hours after completion of antibiotics. He returned 24 hours later with fever of 101.3 Fahrenheit (F). Several blood culture sets were obtained and he was observed off antibiotics for 48 hours during which time he remained afebrile, so he was discharged home. He again returned 24 hours later with fever of $101.9 \mathrm{~F}$. Blood and urine cultures were again performed, his WBC count was 23,600 cells $/ \mathrm{mL}$, and CRP was $3.6 \mathrm{mg} / \mathrm{dL}$. He was observed off antibiotics for 24 hours but the fever curve was increasing so a central venous line $(\mathrm{CVL})$ was placed, new cultures from the line were drawn, and the patient was placed on broad-spectrum antibiotics. The line cultures grew the following day and were identified 
TABLE 1: Blood culture results and susceptibility profile for Staphylococcus epidermidis isolated from the central venous catheter.

\begin{tabular}{lcc}
\hline Antibiotic & MIC & Susceptibility \\
\hline Daptomycin & 0.5 & Susceptible \\
Doxycycline & 1 & Susceptible \\
Erythromycin & $\leq 0.25$ & Susceptible \\
Oxacillin & $\geq 4$ & Resistant \\
Vancomycin & 4 & Susceptible \\
Trimeth-Sulfa & $\leq 10$ & Susceptible \\
\hline
\end{tabular}

MIC = minimum inhibitory concentration, Trimeth-Sulfa $=$ trimethoprimsulfamethoxazole.

as Staphylococcus epidermidis, with a vancomycin minimal inhibitory concentration (MIC) of $4 \mathrm{mcg} / \mathrm{mL}$ (Table 1). Cultures from both CVL lumens were repeated the following day and both grew the same organisms that evening. Vancomycin was continued for 10 days. Repeat cultures remained negative and he remained afebrile and the CVL was removed. Two days after vancomycin was stopped he began to spike fevers again. Daily blood cultures were drawn and were repeatedly sterile. He had normal CBC and CRP values. He remained clinically well and was kept off antibiotics. Possible etiologies were discussed and included infected stents, central fever, and fever related to low cardiac output. We discussed the possibility of culture negative endocarditis; however, he did not meet the major or minor Duke criteria for diagnosis. After much discussion, he was placed on vancomycin 24 hours prior to surgery. He underwent a Glenn procedure and removal of all stents, which were subsequently sent for culture. Cultures from both stents grew bacteria less than 12 hours after removal. The stent from the shunt grew Staphylococcus warneri and Staphylococcus epidermidis. Vancomycin MIC were 1 and $2 \mathrm{mcg} / \mathrm{mL}$, respectively (Table 2). The stent from the atrial septum grew Staphylococcus epidermidis, with a different antibiotic susceptibility profile than the one isolated previously from the CVL and therefore was felt to be a different strain (Table 3). The patient was treated for 6 weeks with intravenous vancomycin and oral rifampin, in combination with gentamicin for the first two weeks. The blood cultures remained negative, and he remained afebrile and clinically well. He was discharged 5 days after antibiotics were completed. He remains symptom-free as documented in a follow-up visit 1 month after discharge.

\section{Discussion}

Fever is a common occurrence in pediatric patients but becomes increasingly cumbersome when the patient is critically ill, after cardiac surgery, or after cardiac catheterization with stent implantation. With the onset of fever, WBC count, CRP or procalcitonin, and blood cultures are typically obtained and empiric antibiotics are commenced. However, in young infants and the early postoperative patient, these inflammatory markers may be unreliable [3]. If cultures remain negative and fever continues without an obvious source of infection, determining the duration of antibiotics becomes more difficult. In cases where there is clinical
TABLE 2: Blood culture results and susceptibility profile for the Staphylococcus epidermidis and Staphylococcus warneri isolated from the stent in the systemic-pulmonary artery shunt.

\begin{tabular}{llcc}
\hline Organism & Antibiotic & MIC & Susceptibility \\
\hline \multirow{4}{*}{ Staph epidermidis } & Daptomycin & 0.5 & Susceptible \\
& Doxycycline & $\geq 16$ & Resistant \\
& Erythromycin & $\leq 0.25$ & Susceptible \\
& Oxacillin & $\geq 4$ & Resistant \\
& Vancomycin & 2 & Susceptible \\
& Gentamicin & $\leq 0.5$ & Susceptible \\
& Rifampin & $\leq 0.5$ & Susceptible \\
\hline & Daptomycin & 1 & Susceptible \\
& Doxycycline & $\leq 0.5$ & Susceptible \\
& Erythromycin & $\geq 8$ & Resistant \\
Staph warneri & Oxacillin & $\leq 0.25$ & Susceptible \\
& Vancomycin & 1 & Susceptible \\
& Gentamicin & $\leq 0.5$ & Susceptible \\
& Rifampin & $\leq 0.5$ & Susceptible \\
\hline
\end{tabular}

MIC = minimum inhibitory concentration.

TABLE 3: Blood culture results and susceptibility profile for Staphylococcus epidermidis isolated from the atrial septal stent.

\begin{tabular}{lcc}
\hline Antibiotic & MIC & Susceptibility \\
\hline Daptomycin & 1 & Susceptible \\
Doxycycline & $\leq 0.5$ & Susceptible \\
Erythromycin & $\geq 8$ & Resistant \\
Oxacillin & $\leq 0.25$ & Susceptible \\
Vancomycin & 1 & Susceptible \\
\hline
\end{tabular}

$\mathrm{MIC}=$ minimum inhibitory concentration.

deterioration or concern, it may be feasible to treat for 710 days, typically with the input of clinical markers and infectious disease guidance.

The above patient is unique due to the persistence of fever and clinical deterioration despite negative blood cultures. He received two separate treatment courses with vancomycin. On both occasions the fever resolved, only to return following antibiotic cessation. He continued with fever for 10 days off antibiotics with daily negative cultures, normal inflammatory markers, and unremarkable physical exam. After much discussion we suspected that the stents were likely colonized/infected and that they should be removed and would do so at the time of the Bidirectional Glenn operation. We also decided that we would monitor the patient closely "off antibiotics" in the hospital with the goal of increasing the culture yield. Per surgical request, vancomycin was commenced 24 hours prior to surgery. As stated above, the cultures from both stents became positive in less than 12 hours. Interestingly, the susceptibility profiles of the bacteria that grew from the stents were different from those that grew from the CVL. Unfortunately, the original Staphylococcus epidermidis isolate was unavailable to perform pulse-field gel electrophoresis, to determine if it was an identical strain. The fevers disappeared following surgery and a prolonged 
antibiotic treatment regimen, similar to the one provided for endocarditis with the presence of prosthetic material.

We believe that this complex infectious disease case is important to report. A recent case series of 85 adults with vascular grafts (the majority for lower limb arterioplasty) found that preoperative blood cultures were positive in only 29 of the 78 patients with an identified organism [4]. In retrospect, the indolent course is consistent with an infection of an endovascular device with a low virulence organism such as coagulase-negative staphylococci. However, his presentation in shock clouded this picture. Our current hypothesis is that the stents were likely seeded following removal of his initial CVL and the infection was festering and did not manifest clinically until after discharge. The present case underscores the need to exclude a stent-associated infection in the setting of persistent fever, normal inflammatory markers, and negative blood cultures and may require surgical extraction for treatment.

\section{Conflict of Interests}

The authors declare that they have no conflict of interests regarding the publication of this paper.

\section{References}

[1] M. A. Villasís-Keever, D. M. Zapata-Arenas, and M. J. PenagosPaniagua, "Frequency of postoperative fever in children with congenital heart disease undergoing cardiovascular surgery and associated risk factors," Revista Espanola de Cardiologia, vol. 55, no. 10, pp. 1063-1069, 2002.

[2] R. Dayal and D. Agarwal, "Fever in children and fever of unknown origin," Indian Journal of Pediatrics, In press.

[3] P. McMaster, D. Y. Park, F. Shann et al., "Procalcitonin versus C-reactive protein and immature-to-total neutrophil ratio as markers of infection after cardiopulmonary bypass in children," Pediatric Critical Care Medicine, vol. 10, no. 2, pp. 217-221, 2009.

[4] L. Legout, B. Sarraz-Bournet, P. V. D'Elia et al., "Characteristics and prognosis in patients with prosthetic vascular graft infection: a prospective observational cohort study," Clinical Microbiology and Infection, vol. 18, no. 4, pp. 352-358, 2012. 


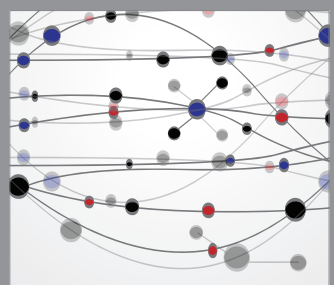

The Scientific World Journal
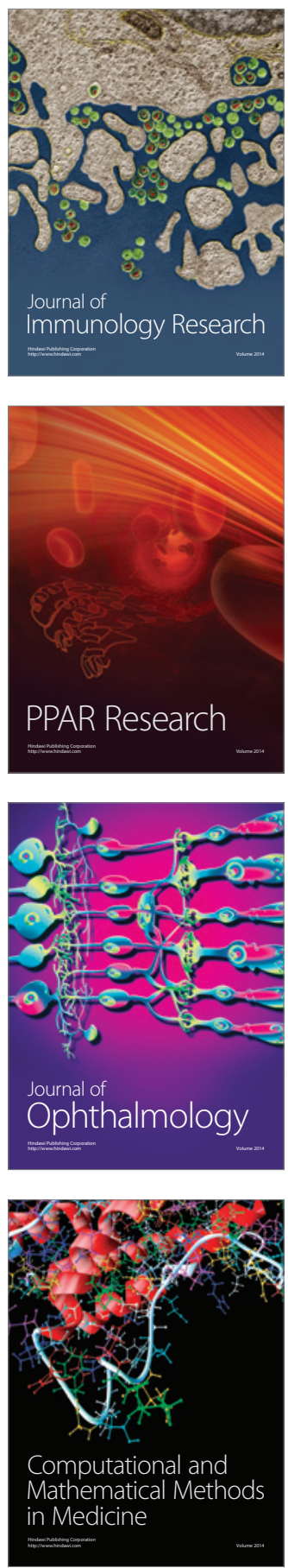

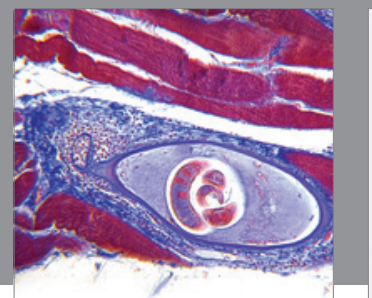

Gastroenterology

Research and Practice
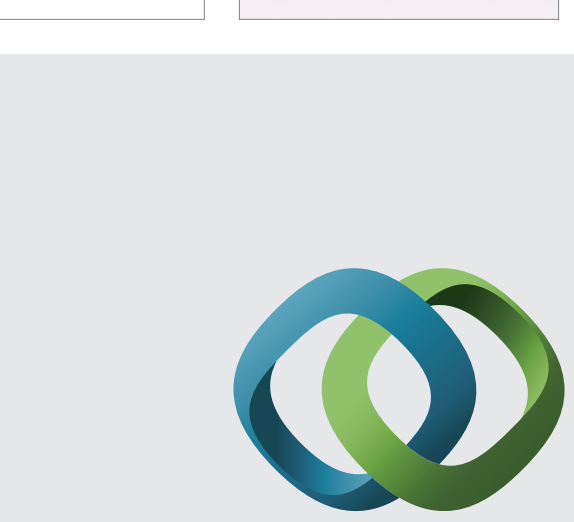

\section{Hindawi}

Submit your manuscripts at

http://www.hindawi.com
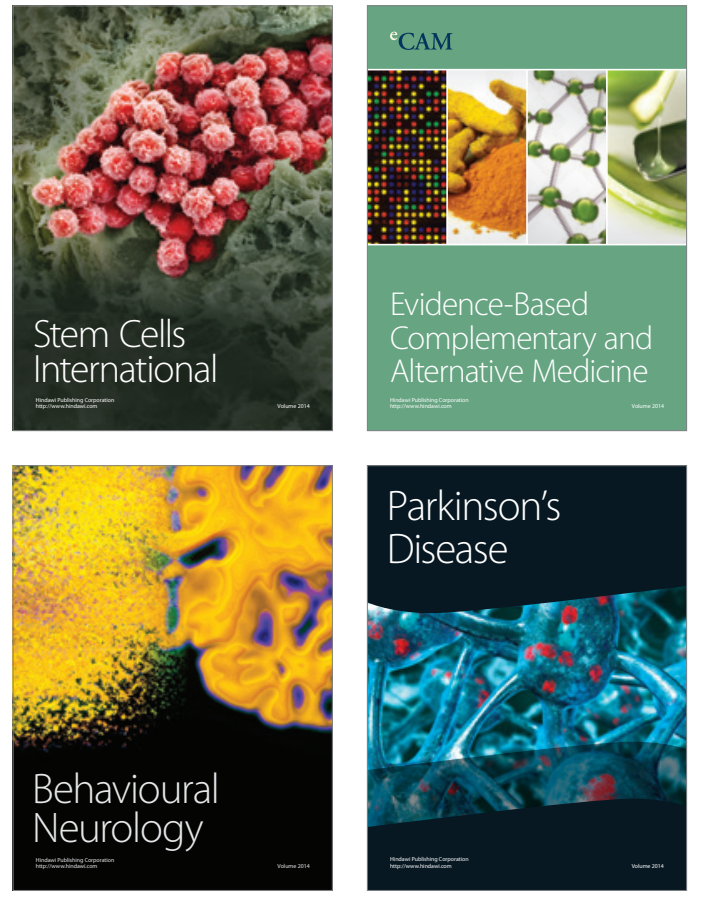
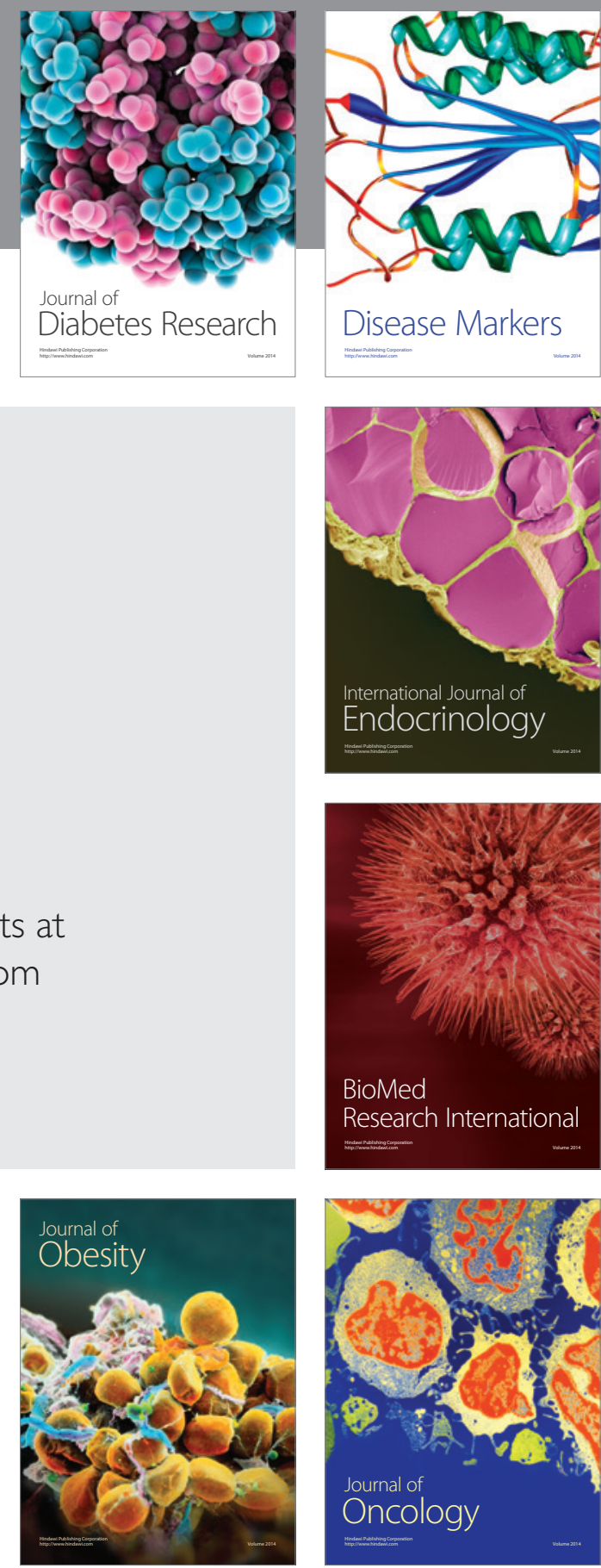

Disease Markers
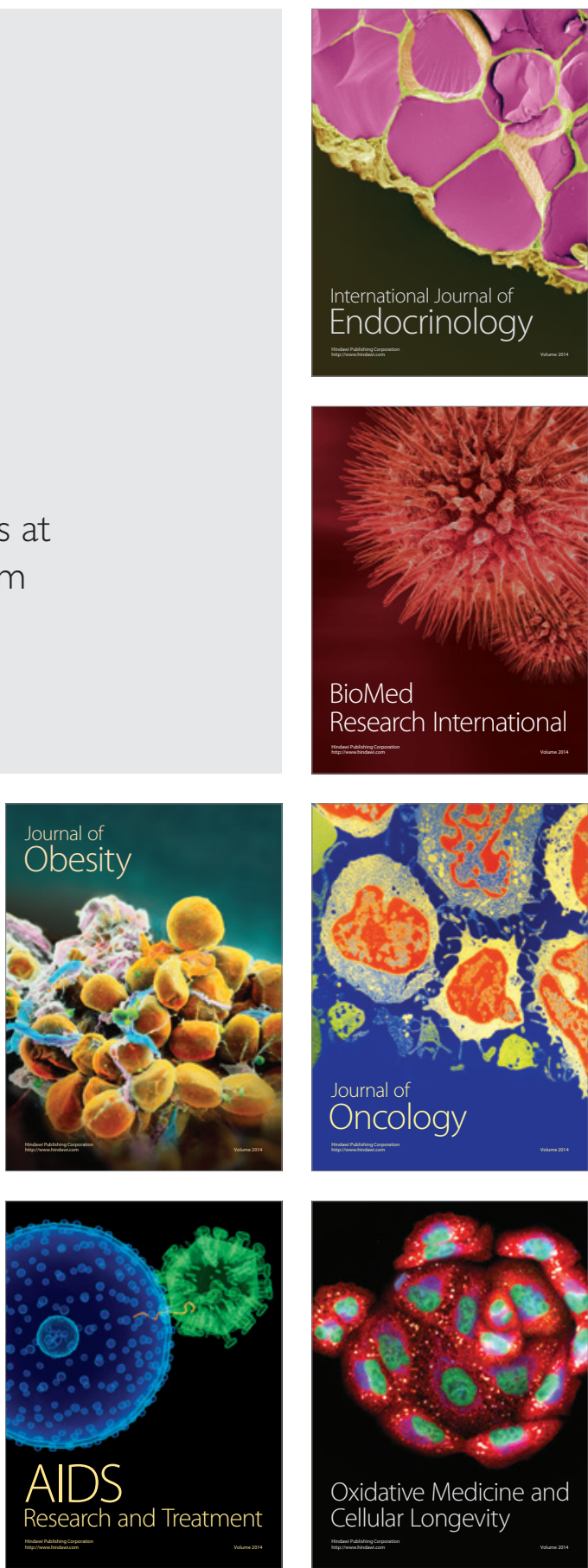